\title{
Reduction of Reconstruction Errors in Kinoform CGHs by Modified Simulated Annealing Algorithm
}

\author{
Han-Jin Yang, Jeong-Sik Cho*, and Yong-Hyub Won \\ School of Engineering, Information and Communication University, Daejeon 305-732, Korea
}

(Received January 5, 2009 : revised March 2, 2009 : accepted March 2, 2009)

\begin{abstract}
In this paper, a conventional simulated annealing (SA) method for optimization of a kinoform computer generated hologram $(\mathrm{CGH})$ is analyzed and the SA method is modified to reduce a reconstruction error rate (ER) of the CGH. The dependences of the quantization level of the hologram pattern and the size of the data on the ER are analyzed. To overcome saturation of the ER, the conventional SA method is modified as it magnifies a Fourier-transformed pattern in the intermediate step. The proposed method can achieve a small ER less than 1\%, which is impossible in the conventional SA method.
\end{abstract}

Keywords : Hologram identification, Kinoform, Simulated annealing, Computer generated hologram

OCIS codes: (040.5160) Holography; (100.0100) Image processing; (210.0210) Optical data storage

\section{INTRODUCTION}

A computer generated hologram (CGH) [1] inherits the outstanding properties of a hologram such as phase recording, anti-forgery, simultaneous recording of a whole image, and so on, so it has been used to develop a holographic data storage system [2], three-dimensional display [3], digital hologram label [4], optical elements [5,6], etc. One application, the storage technology of a digital hologram in a thin medium, has recently been found attractive because of its potential of application to an identification (ID) system [7]. In this paper, we focus on how to generate the digital hologram pattern for hologram ID applications. In the applications, the hologram pattern is written by direct sculpturing by a laser rather than by two-beam interference used in the conventional scheme $[2,7]$. This approach enables fast fabrication of a hologram ID tag and provides flexibility for the storage application of the hologram ID. However, choosing the laserbased fabrication scheme results in restrictions in simultaneous representation of the phase and amplitude information of a hologram. To meet these requirements, we choose the kinoform technology [8] as a representation method of the CGH pattern in the hologram ID tag.

The kinoform can be used in many applications inclu-

*Corresponding author: jscho@icu.ac.kr ding optical information processing [9], optical interconnection [5], and spatial filtering [10], owing to its high diffraction efficiency and its flexibility of design. The kinoform is a phase-only optical element generated by a computer, so the amplitude of the transfer function of the kinoform is assumed to be constant. The maximum phase modulation in the kinoform is $2 \pi$. The phase information, consisting of ordinary quantized values, is usually represented as a relief profile on a recording material such as a photographic emulsion or is written on a spatial light modulator as a phase modulator. The kinoform could include reconstruction noises caused by neglect of the amplitude information and quantization of the phase information [11,12]. Because reconstruction noise is a serious problem in many applications, the phase distribution of the kinoform needs to be optimized to reduce the noise. Several methods [13-16] have been proposed for phase optimization of the kinoform. One of them, the simulated annealing (SA) has been considered as an effective method to avoid the trap of a local minimum [16]. However, its accuracy is not good enough to be accepted and its computation time is impractically long.

In this paper, the validity of the kinoform CGH synthesized by the SA method for hologram data storage is analyzed and discussed. We discuss the relation between an initial temperature in the SA algorithm 
and the convergence speed to the global minimum of a cost function. We also analyze the efficient quantization level of the kinoform CGH. We investigate the dependence of a reconstruction error rate on the size of hologram data. Finally, we propose modification of the optimization algorithm that greatly reduces the reconstruction error rate, which encourages effective representation of a Fourier transformed hologram pattern using a magnification method. All of the simulation works in this paper have been performed by using a computing environment of $2.8 \mathrm{GHz}$ Pentium $\mathrm{D}^{\mathrm{TM}} \mathrm{CPU}$ and $2 \mathrm{~GB}$ RAM.

\section{KINOFORM CGH OPTIMIZATION BY SIMULATED ANNEALING}

The SA method optimizes the phase distribution of a kinoform CGH to reduce the reconstruction noise of the CGH pattern [16]. Fig. 1 shows the conventional SA algorithm. The optimized point is acquired by reducing the value of the cost function, $E(\theta)$. To find the global minimum of the cost function efficiently, an initial value of the temperature parameter must be selected deliberately. The perturbation probability, $P(\Delta E)=\exp (-\Delta E / T)$, which ensures finding the global minimum of the cost function, is strongly dependent on the initial value of the temperature parameter. The temperature parameter does not mean a real temperature like Kelvin or Celsius degrees. In general, it is known that if we choose a high temperature and cool the temperature down at a slow rate, then the cost function could not be trapped in a local minimum. However, the high temperature results in a long optimization time to achieve a reasonable level of the reconstruction error rate because the acceptance probability is so high for the same $\Delta E$ and the number of processes to accept the phase changed state as appropriate for finding the global minimum increases greatly. When too low an initial temperature is used, the acceptance probability becomes low for the same $\Delta E$. In this case, many cases of state change could be neglected. So the time to reach the global minimum of the cost function could increase. Here we empirically choose 100 as the initial temperature value. Fig. 2 shows an original two-dimensional image of the $49 \times 49$ data size used throughout this paper, and cost functions for different initial temperatures.

Figure 3 shows how to retrieve the data from the two-dimensional hologram pattern. When a coherent light beam illuminates the hologram pattern, a reflected light will reach a charge coupled device (CCD) camera through a Fourier lens, which is located between the hologram pattern and the CCD camera and carries out optical Fourier transform of the passing light. This reconstruction scheme has already been considered in the kinoform SA algorithm. The reconstructed data do not

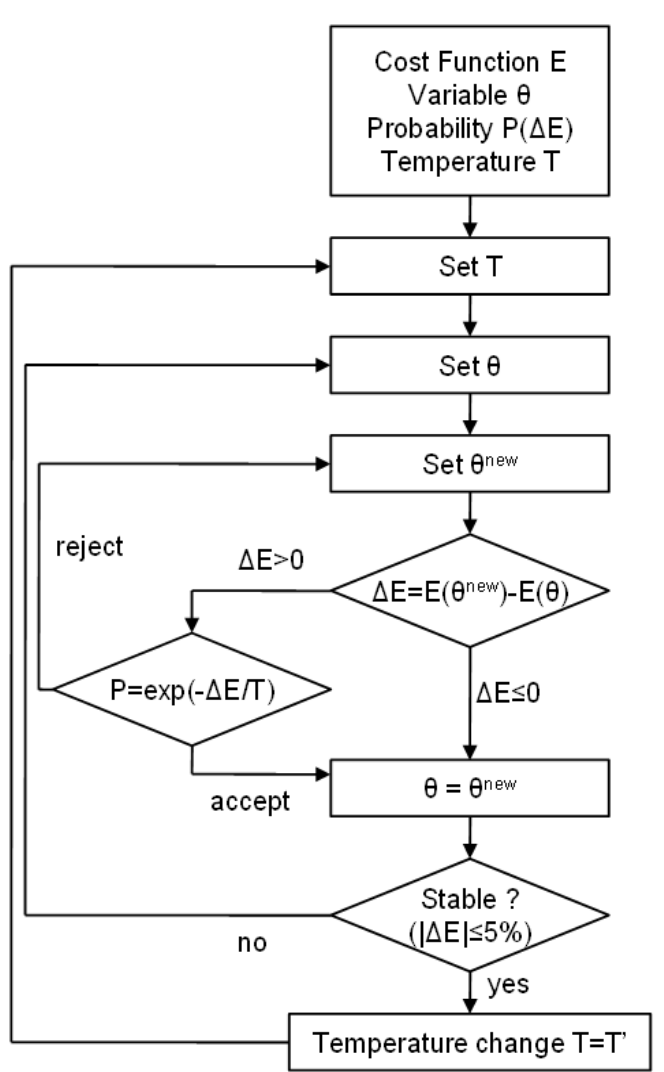

FIG. 1. Algorithm of the conventional SA method.

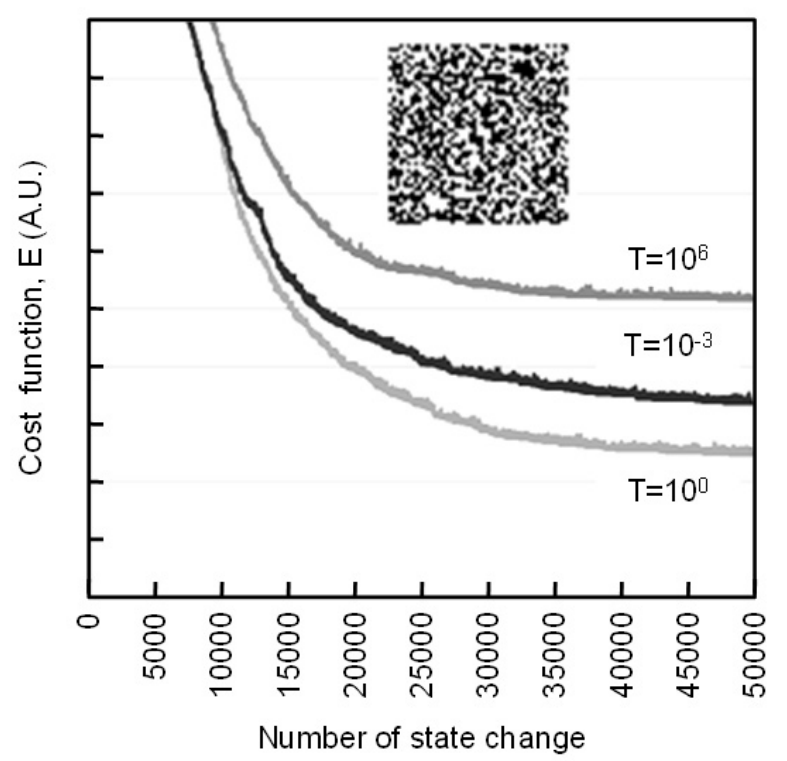

FIG. 2. Cost function versus stage change number for different initial temperatures. Inset: $49 \times 49$ data image.

perfectly match with the original data because the reconstructed hologram pattern has noise due to neglect of the amplitude information and quantization of the phase information. The SA method reduces the reconstruction noise by remapping the phase distribution. 


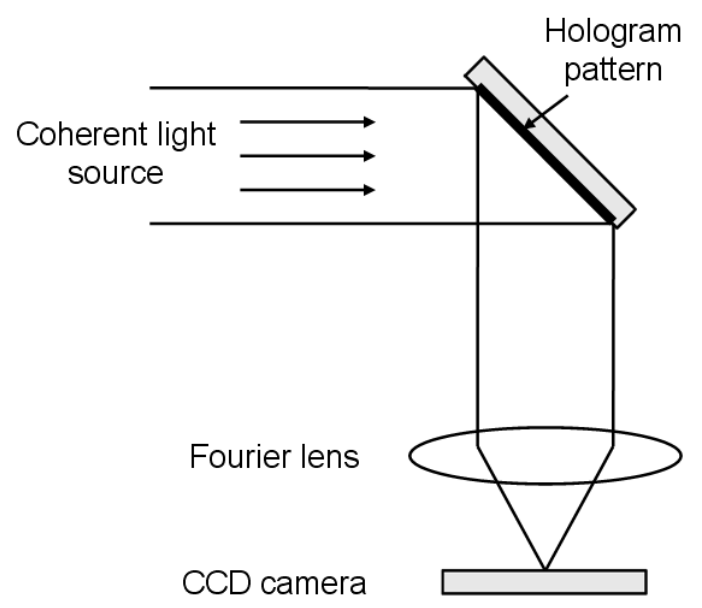

FIG. 3. Schematic diagram for data reproduction from the hologram pattern.

After the SA process, all the reconstructed intensities are quantized into two levels ' 0 ' and ' 1 ', by applying a predetermined threshold value which maximizes the agreement with the original data. The agreement can be measured by an error rate defined as,

$$
E R=\frac{\text { number of error pixel }}{\text { data size }} \times 100(\%)
$$

where the data size is the total number of pixels. Smaller ER means better quality of reconstruction.

\section{ANALYSIS OF KINOFORM CGH AND MODIFIED SA ALGORITHM}

\section{Quantization of the pixel amplitude}

Figure 4 shows that the ER saturates as the iteration number increases in every quantization level of kinoform representation. The iteration number means the number of temperature cooling down steps after acceptance of the change of the cost function. After the ER reaches the saturation value, the pattern generation time increases abruptly. It means that the reconstructed pattern is fully optimized so that further change of the state cannot improve the reconstruction ER. Fig. 4(a-f) shows that the ER saturates at an ER of $23 \%$ in 3-level, $14 \%$ in 5-level, $12 \%$ in 7-level, $11 \%$ in 9-level, $9 \%$ in 11-level, and $9 \%$ in 13 -level. The ER could be maximally decreased to $9 \%$ even in the saturation state of the 11 quantization level. So we concluded that the 11-level representation is the most efficient quantization condition and $9 \%$ is the lowest ER that we can achieve in a reasonable pattern generating time.

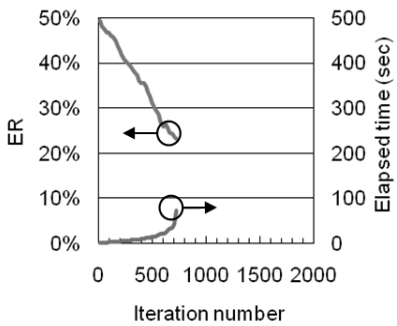

(a)

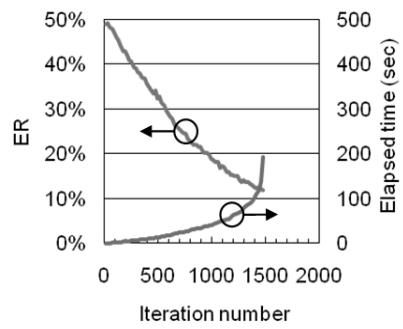

(c)

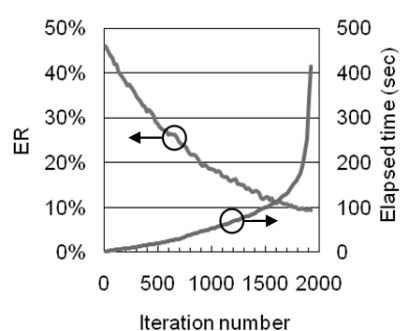

(e)

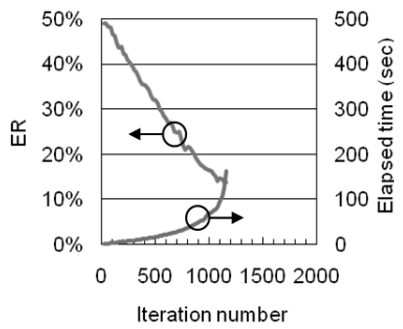

(b)

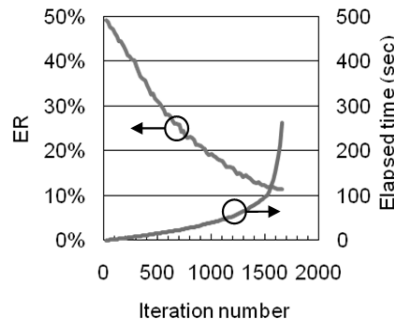

(d)

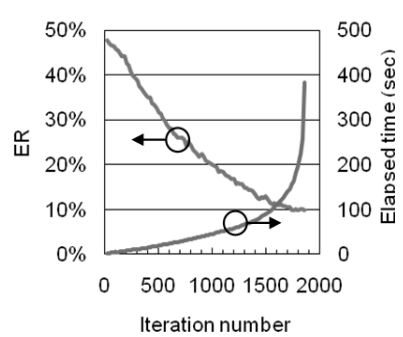

(f)
FIG. 4. ER and elapsed time according to the quantization level of (a) 3, (b) 5, (c) 7, (d) 9, (e) 11, and (f) 13.

\section{Size of hologram data}

Figure 5 shows the ER of 11-level kinoform representation of $30 \times 30,50 \times 50,70 \times 70$ and $90 \times 90$ data sizes, and it shows that the converged ER values have no tight relations with the data size. As shown in Fig. 4, the quantization level of kinoform representation is the key factor to determine the saturated ER. The iteration number of the converged $\mathrm{ER}$ value increases as the data size increases. Because the iteration number is basically proportional to the elapsed time for generation, the pattern generating time to have a specific ER also can be expected to increase as the data size increases.

When the stored data is reproduced from the two dimensional hologram, a predetermined threshold value must be applied to the captured image data to determine digital value ' 1 ' or ' 0 '. Usually the threshold value is half of the maximum amplitude of all pixels. However, we observed that the optimized threshold levels for different data sizes increases as the data size increases as shown in Fig. 6. Average intensity values of the captured image were 25 out of 255 maximum for $30 \times 30,40$ for $50 \times 50,55$ for $70 \times 70$, and 70 for $90 \times 90$ data sizes, and they were used to calculate the matched threshold levels of $10 \%$, 
$16 \%, 22 \%$ and $27 \%$. In the hologram pattern, there always exists a direct current spot noise in the center of the image due to the Fourier transform process. It has a maximum value all the time and its bandwidth is very narrow. Thus its relative weight in the small data size condition becomes large, that is, it heavily influences the overall intensity sum. We observed that the brightness of each pattern increases as the pattern size increases, as shown in Fig. 6.

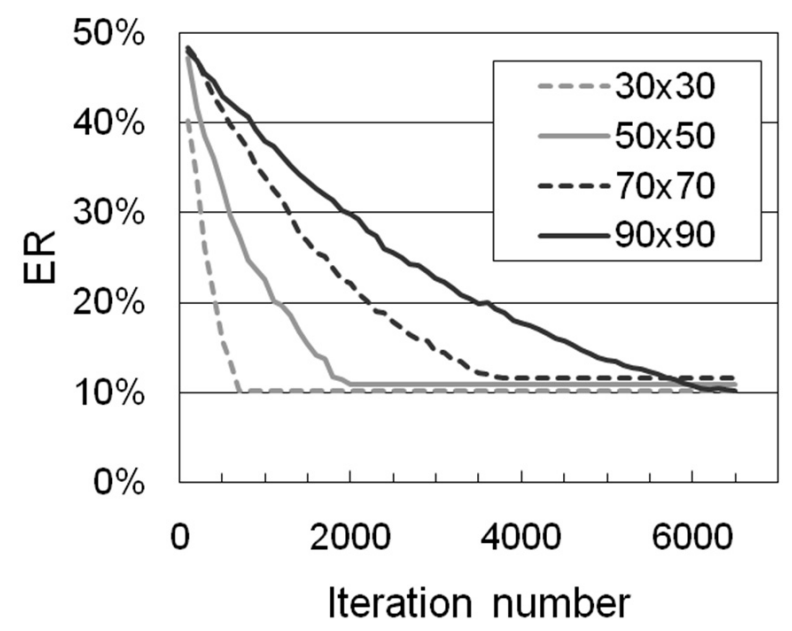

FIG. 5. ER measurement for different data sizes at the 11 quantization level.

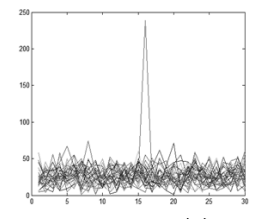

(a)

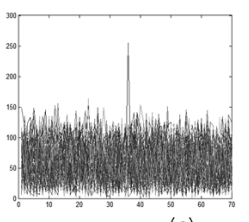

(c)
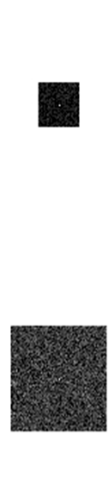

$50 \%$

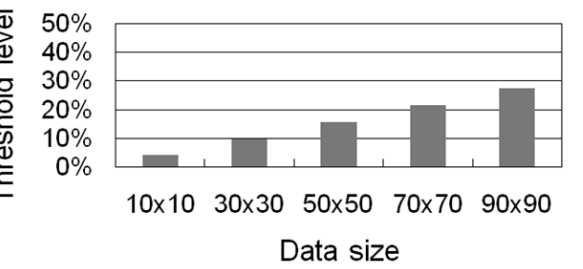

(e)

FIG. 6. Side views (left) and top views (right) of intensity distribution of captured images for different data sizes of (a) $30 \times 30$, (b) $50 \times 50$, (c) $70 \times 70$, and (d) $90 \times 90$. (e) Optimized threshold levels for different data sizes.

\section{Modified SA algorithm}

The conventional SA method cannot reduce the ER down below around $10 \%$ in an acceptably long pattern generating time. In the simulation, even though we considered a large generating time, i.e. decades of minutes, we achieved near $9 \%$ ER at most. To overcome this limitation, we proposed modification of the SA algorithm. Fig. 7 shows the modified algorithm. In the conventional SA method, the size of the hologram pattern is exactly the same as the original data size. But in the newly proposed method, the size of the Fouriertransformed hologram pattern generated in the intermediate step is set to be bigger than the original data size in order to represent the phase information in high resolution. The modified algorithm helps the reduction of the quantization errors induced by two-dimensional digital representation of the fine phase information of the Fourier transformed image. The reconstructed data size is also larger than the original data size. Thus the reconstructed image must be reduced to be compared with the original image. The pixel intensity of the final image should be redefined as follows,

$$
I(x, y)=\frac{\sum_{k=0}^{n-1} \sum_{l=0}^{n-1} I_{m a g}(n \cdot x-k, n \cdot y-l)}{n^{2}}
$$

where $I_{\text {mag }}(x, y)$ is the intensity of the magnified image at $(\mathrm{x}, \mathrm{y})$ coordinate and $n$ is a magnification factor. The intensity of the reconstructed image, $I(x, y)$, is compared with the original intensity, $I_{0}(x, y)$, to estimate the ER. All other procedures of the phase optimization of the kinoform CGH are the same with the conventional SA method.

Figure 8 shows the improved ER using the proposed method. In the simulation, the magnification factor $n$ was 4 . The results are summarized in Table 1.

We estimated the ER until maximally 452 minutes, i.e. seven and half hours. But we could not find any

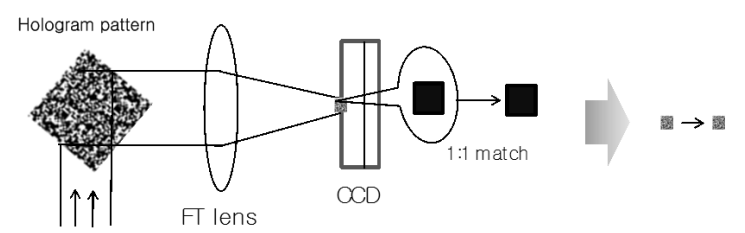

(a)

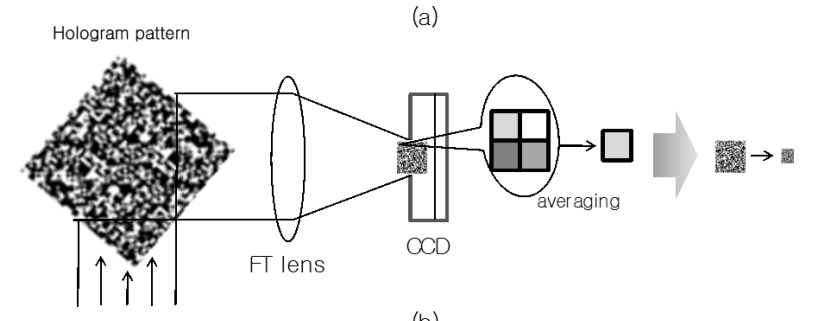

(b)

FIG. 7. Data reconstructions in (a) the conventional SA method and (b) the proposed SA method. 
abrupt increase in the pattern generation time, which happened when the previous algorithm was applied, except for the 3-level kinoform representation case. Even in the 3-level representation case, the abruptness was considerably mitigated compared to that of the conventional SA algorithm case. If we consume more time to optimize the hologram pattern, then we can obtain slightly improved results. But the seven and half hours already can be considered as a meaningless or unacceptable time for pattern generation purposes. So the results in Table 1 could be considered as actual saturation values of the ER.

In the proposed SA method, when the phase of one

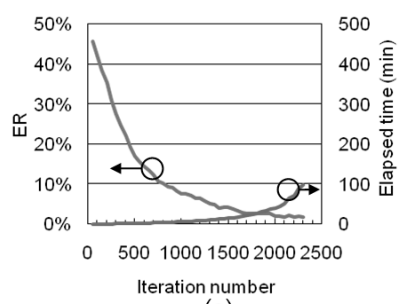

(a)

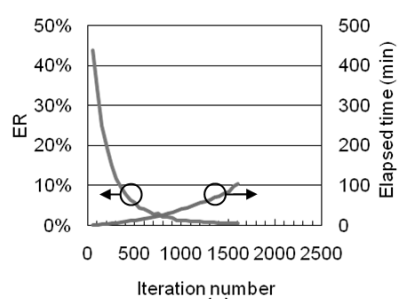

(c)

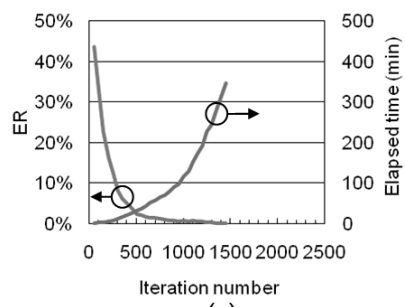

(e)

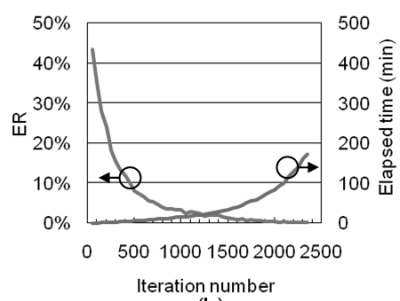

(b)

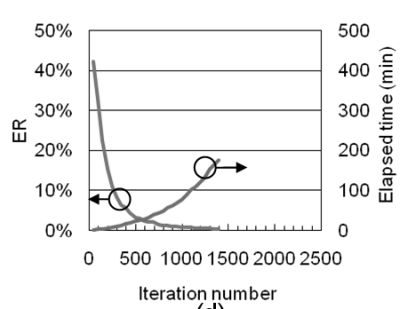

(d)

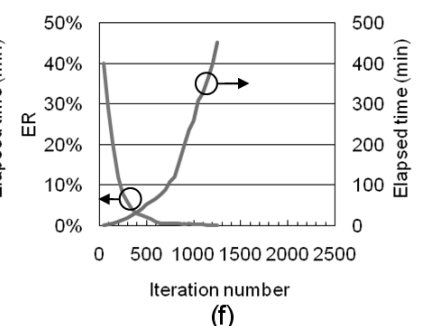

(f)
FIG. 8. ER and elapsed time for the quantization level of (a) 3, (b) 5, (c) 7, (d) 9, (e) 11, and (f) 13 by the proposed SA method.

TABLE 1. ERs by the proposed and conventional methods for quantization levels.

\begin{tabular}{c|c|c}
\hline \hline $\begin{array}{c}\text { Quantization } \\
\text { level }\end{array}$ & $\begin{array}{c}\text { ER by proposed } \\
\text { method }\end{array}$ & $\begin{array}{c}\text { ER by conventional } \\
\text { method }\end{array}$ \\
\hline 3 & 1.8 & 23 \\
\hline 5 & 0.25 & 14 \\
\hline 7 & 0.25 & 12 \\
\hline 9 & 0.25 & 11 \\
\hline 11 & 0.21 & 9 \\
\hline 13 & 0.17 & 9 \\
\hline
\end{tabular}

pixel of the hologram pattern is changed, its effect would be relieved because the pixel's phase information is averaged with adjacent pixels. Simultaneous phase change of a couple of pixels is worth a try. The results are shown in Fig. 9. When one pixel was changed, the ER saturated around $10 \%$. The ER was improved when we chose a two pixel change scheme. Then the performance was getting worse as the pixel change number increased. When a couple of pixels were changed simultaneously, the hologram pattern could approach the optimized state faster. But when too many pixels are flipped simultaneously, the cost function fluctuates a lot and, even, there is a probability of change of the same pixel with what was changed in the previous step. Thus the two-pixel change scheme is the best in the proposed method and we can obtain less than $1 \%$ ER. If we run the simulation for a long time, then we can further reduce the ER. However, the elapsed time turns out to be unacceptably long. Thus, in the following study, this must be improved for commercialization of the proposed method.

Using the proposed SA method, we analyzed the pattern generation time to achieve a specific ER. Fig. 10 shows the results for the $2 \%$ and $1 \%$ ER cases. The pattern generation time increases as the quantization level increases. In the case of 3-level quantization, the ER saturates at $1.8 \%$. So we concluded that 5 -level quantization of the kinoform CGH is the most optimized condition in the proposed method.

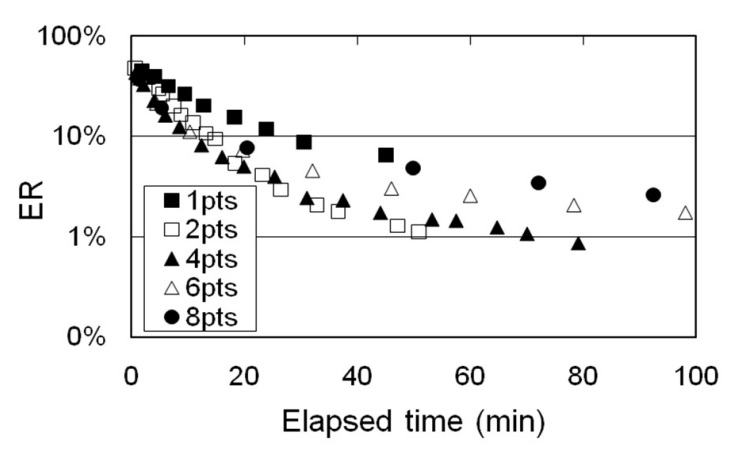

FIG. 9. ER and elapsed time for different number of flipped pixels.

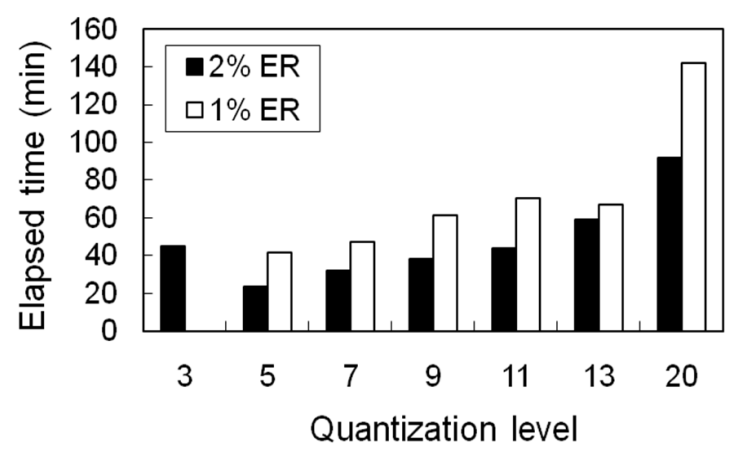

FIG. 10. Pattern generation time to reach $2 \%$ and $1 \%$ ER. 


\section{CONCLUSION}

We have analyzed the conventional SA method for optimization of the kinoform CGH and proposed the modified SA method to reduce the ER of the reconstructed data. We observed the temperature dependence of the cost function and chose a proper initial temperature. Effect of quantization of pixel intensity of the hologram image on the ER of the reconstructed data was analyzed, and saturation of the ER was observed. Independence of the data size on the ER saturation was also observed. To overcome the limit in the ER, modification of the SA algorithm was proposed. The modified SA method magnifies the Fourier-transformed image that is temporarily generated in the optimization process to reduce the noise induced by digital representation of the phase information of the Fourier-transformed image. Using the proposed method, we could obtain a small enough ER of less than $1 \%$. The discussions and proposed method can be used for development of the hologram ID system.

\section{ACKNOWLEDGMENT}

This work was supported by the IT R\&D program of MKE/IITA.[2008-F-049-01, Development of Optical ID Technology]

\section{REFERENCES}

1. W. H. Lee, "Binary computer-generated holograms," Appl. Opt. 18, 3661-3669 (1979).

2. J. Ashley, M.-P Bernal, G. W. Burr, H. Coufal, H. Guenther, J. A. Hoffnagle, C. M. Jefferson, B. Marcus, R. M. Macfarlane, R. M. Shelby, and G. T. Sincerbox, "Holographic data storage," IBM J. Res. Develop. 44, 341-368 (2000).

3. T.-C. Poon Ed., Digital Holography and Three-Dimensional
Display: Principles and Applications (Springer, Berlin, Germany, 2006).

4. N. Takahashi and T. Fujiyoshi, "System and process for reading hologram code, hologram and card containing hologram," U.S. Patent 5444225 (1995).

5. M. P. Dames, R. J. Dowling, P. McKee, and D. Wood, "Efficient optical elements to generate intensity weighted spot arrays: design and fabrication," Appl. Opt. 30, 2685-2691 (1991).

6. A. Cable, P. Mesh, and T. Wilkinson, "Production of computer-generated holograms on recordable compact disk media using a compact disk writer," Opt. Eng. 42, 2514-2520 (2003).

7. J. Kim, J. Choi, J. An, N. Kim, and K. Lee, "Digital holographic security system based on random phase encoded reference beams and fingerprint identification," Opt. Comm. 247, 265-274 (2005).

8. L. B. Lesem, P. M. Hirsch, and J. A. Jordan, Jr., "The kinoform: a new wavefront reconstruction device," IBM J. Res. Develop. 13, 150-155 (1969).

9. Y. Hamano and H. Hirai, "Optical pickup and optical information processing apparatus," PCT Patent, WO2007/ 123250 (2007).

10. A. Kolodziejczyk, Z. Jaroszewiczb, A. Kowalikc, and O. Quinterod, "Kinoform sampling filter," Opt. Comm. 200, 35-42 (2001).

11. M. C. Gallagher and B. Lui, "Method for computing kinoforms that reduces image reconstruction error," Appl. Opt. 12, 2328-2335 (1973).

12. T. Yatagai and M. Takeda, "Effect of phase nonlinearity in kinoform," Optik 43, 337-352 (1975).

13. J. R. Fienup, "Iterative method applied to image reconstruction and to computer-generated holograms," Opt. Eng. 19, 297-305 (1980).

14. F. Wyrowski and O. Bryngdahl, "Iterative Fouriertransform algorithm applied to computer holography," J. Opt. Soc. Am. 5, 1058-1065 (1988).

15. M. A. Seldowitz, J. P. Allebach, and D. W. Sweeney, "Synthesis of digital holograms by direct binary search," Appl. Opt. 26, 2788-2798 (1987).

16. N. Yoshikawa and T. Yatagai, "Phase optimization of a kinoform by SA," Appl. Opt 33, 863-868 (1994). 